\title{
Origin of the superconductivity in the Y-Sr-Ru-O and Y-Sr-Cu-O systems
}

\author{
Eduard Galstyan, ${ }^{1}$ Yuyi Xue, ${ }^{1}$ Milko Iliev, ${ }^{1}$ Yanyi Sun, ${ }^{1}$ and Ching-Wu Chu ${ }^{1,2,3}$ \\ ${ }^{1}$ Department of Physics and Texas Center for Superconductivity, University of Houston, Houston, Texas 77204-5002, USA \\ ${ }^{2}$ Lawrence Berkeley National Laboratory, 1 Cyclotron Road, Berkeley, California 94720, USA \\ ${ }^{3}$ Hong Kong University of Science and Technology, Hong Kong, China \\ (Received 16 April 2007; published 3 July 2007)
}

\begin{abstract}
We report on the structural, magnetic, and Raman-scattering studies of double-perovskite structure $\mathrm{Sr}_{2} \mathrm{YRu}_{1-x} \mathrm{Cu}_{x} \mathrm{O}_{6-\delta}$ systems made by systematic synthesis processes with various numbers of doping concentrations and sintering temperatures. We observed different behaviors resulting from the different thermal treatments. In particular, superconductivity in Cu-doped $\mathrm{Sr}_{2} \mathrm{YRuO}_{6}$ has been observed only for partially melted ceramic materials. We show that superconductivity is associated with the 1:2:3 phase $\left(\mathrm{YSr}_{2} \mathrm{Cu}_{3} \mathrm{O}_{t}\right)$, similar to that of Y-Sr-Cu-O samples sintered at high temperature.
\end{abstract}

DOI: 10.1103/PhysRevB.76.014501

PACS number(s): 74.62.Bf, 74.70.Pq, 74.72.-h, 74.72.Bk

\section{INTRODUCTION}

The nature and origin of the superconductivity (SC) in perovskite-based cuprates have attracted much interest among researchers in recent years. The common features of the high-temperature superconducting cuprates are sequences of $\mathrm{CuO}_{2}$ layers that upon sufficient doping become superconducting. It has been one major challenge to search for the possibility of finding other superconductors that contain no $\mathrm{Cu}-\mathrm{O}$ planes. Initially, this interest was caused by the discovery of exotic SC in $\mathrm{Sr}_{2} \mathrm{RuO}_{4}{ }^{1}{ }^{1}$ Later, it was found that other ruthenates have very interesting magnetic and electric properties. Among them, there has been particular interest in the Ru-based double-perovskite structure $\mathrm{Sr}_{2} \mathrm{YRuO}_{6}$ systems with minor $\mathrm{Cu}$ doping on $\mathrm{Ru}$ ion sites. ${ }^{2-5}$ The parent insulator compounds $\mathrm{Sr}_{2} \mathrm{YRuO}_{6}$ can be obtained from the itinerant ferromagnet $\mathrm{SrRuO}_{3}$ by replacing each second $\mathrm{Ru}$ ion with a nonmagnetic $\mathrm{Y}$ ion. ${ }^{6-9}$ The antiferromagnetic (AFM) transition temperature $\left(T_{N}\right)$ is $26 \mathrm{~K}$ and there is a spin-flop-like transition below $T_{M} \approx 17 \mathrm{~K} .^{8}$ An effective paramagnetic moment $\mu_{e f f}$ was found to be $3.13 \mu_{B} / \mathrm{Ru}^{5+}$, which was attributed a spin-orbit coupling. ${ }^{7}$ Below $T_{N}=26 \mathrm{~K}$, the facecentered cubic lattice of $\mathrm{Ru}^{5+}$ spins experiences ordering to produce a type-I AFM structure with a weak ferromagnetic component. ${ }^{8,9}$ In this structure, the (001) ferromagnetic (FM) planes exhibit antiparallel ordering along the $c$ axis. Intriguingly, $\mathrm{SC}$ has been reported in $\mathrm{Sr}_{2} \mathrm{YRu}_{1-x} \mathrm{Cu}_{x} \mathrm{O}_{6-\delta}$ ceramic materials with an onset transition temperature of $T_{C}$ $\approx 45-49 \mathrm{~K}$ for $x=0.03-0.50 .^{2-5}$ Addressing the question of the nature of SC, Dow and Harshman ${ }^{5,10,11}$ proposed a model in which superconducting condensation occurs in the $\mathrm{SrO}$ planes with the $\left(\mathrm{Ru}_{1-x} \mathrm{Cu}_{x}\right) \mathrm{O}$ planes magnetically ordered. While the model appears to be in disagreement with many research works, ${ }^{12-16}$ the exact reason for SC in Y-Sr-Ru-O system is still uncertain. In order to gain additional insight into the superconducting properties of $\mathrm{Cu}$-doped $\mathrm{Sr}_{2} \mathrm{YRuO}_{6}$ ceramic compounds, we present the results of magnetic measurements and Raman-scattering investigation. The crystal structure, lattice parameters, and detailed microstructure were analyzed by $\mathrm{x}$-ray powder diffraction (XRD) method and scanning electron microscopy (SEM). In this work, we provide experimental evidence that the SC behavior of these materials is due to a minor impurity phase with the stoichiometry of $\mathrm{YSr}_{2} \mathrm{Cu}_{3} \mathrm{O}_{t}(\mathrm{YSCO})$. We show that both superconductivity and YSCO grains appear only for synthesis temperatures higher than a local-melting temperature identifiable by the differential thermal analyzer (DTA) trace. In addition, the YSCO grains, which are thermodynamically unstable under ambient pressure, appear within dense surroundings, where the melting indications are obvious. The stresses during the solidification seem to play roles. These may also explain the earlier reports of trace $\mathrm{SC}$ in $\mathrm{Y}-\mathrm{Sr}-\mathrm{Cu}-\mathrm{O}$ samples synthesized at ambient pressure. ${ }^{17,18}$

\section{EXPERIMENTAL DETAILS}

Ceramic samples with nominal compositions $\mathrm{Sr}_{2} \mathrm{YRu}_{1-x} \mathrm{Cu}_{x} \mathrm{O}_{6-\delta} \quad(x=0.0,0.1,0.5) \quad$ and $\quad \mathrm{Y}-\mathrm{Sr}-\mathrm{Cu}-\mathrm{O}$ $\left(\mathrm{YSrCuO}_{t}, \mathrm{YSr}_{2} \mathrm{Cu}_{2} \mathrm{O}_{t}, \mathrm{Y}_{1.5} \mathrm{Sr}_{2} \mathrm{Cu}_{2} \mathrm{O}_{t}\right.$ ) were prepared by a solid-state reaction technique. Prescribed amounts of $\mathrm{Y}_{2} \mathrm{O}_{3}$, $\mathrm{SrCO}_{2}, \mathrm{RuO}_{2}$, and $\mathrm{CuO}$ were mixed, pressed into pellets, and preheated at $1000{ }^{\circ} \mathrm{C}$ for 1 day $\left(850{ }^{\circ} \mathrm{C}\right.$ for $\left.\mathrm{Y}-\mathrm{Sr}-\mathrm{Cu}-\mathrm{O}\right)$. The first and second batches of $\mathrm{Sr}_{2} \mathrm{YRu}_{1-x} \mathrm{Cu}_{x} \mathrm{O}_{6-\delta}$ were cooled, reground, and sintered under an oxygen atmosphere for 1 day at 1290 and $1360{ }^{\circ} \mathrm{C}$, respectively, and finally the samples were slowly cooled at $15^{\circ} \mathrm{C} / \mathrm{h}$ to room temperature. Some of the Y-Sr-Cu-O calcinated powders were pelletized and heated at $990{ }^{\circ} \mathrm{C}$ for 3 days under ambient pressure in an oxygen atmosphere. Other $\mathrm{Y}-\mathrm{Sr}-\mathrm{Cu}-\mathrm{O}$ calcinated materials were reheated at $1130{ }^{\circ} \mathrm{C}$ for $6 \mathrm{~h}$ in flowing oxygen. XRD was used to identify the bulk phase, approximate composition, and lattice parameters. Diffraction patterns were obtained with $\mathrm{Cu} K \alpha$ radiation (wavelength $\lambda$ $=0.154178 \mathrm{~nm})$ over a range of $15^{\circ}<2 \theta<80^{\circ}$ using $0.04^{\circ}$ steps. The microstructure and the phase integrity of the materials were investigated by SEM and by a Genesis energy dispersive $\mathrm{x}$-ray analysis (EDAX) device attached to the SEM. The melting temperature was determined with a DTA. Magnetic susceptibility data were obtained with a superconducting quantum interference device magnetometer. Polarized Raman spectra were collected under microscope (focus spot size 1-3 $\mu \mathrm{m}, \lambda_{e x c}=488 \mathrm{~nm}$ ) from the polished surface of the bulk materials at room temperature. 


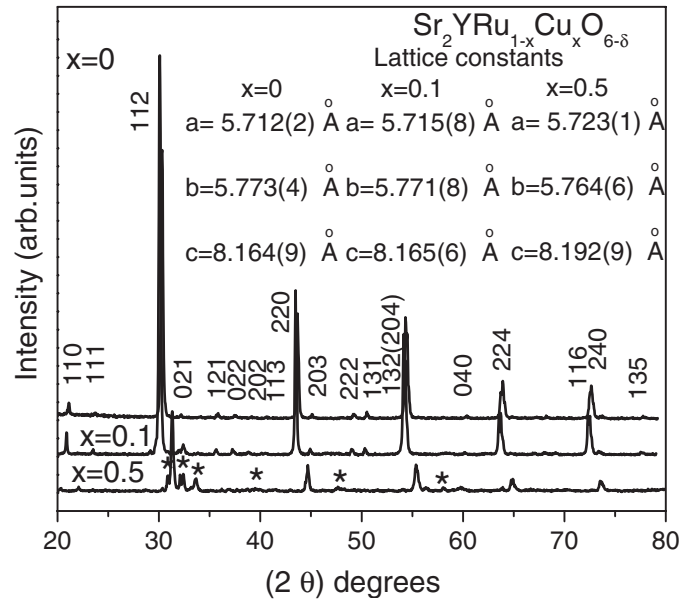

FIG. 1. Powder $\mathrm{x}$-ray diffraction patterns of $\mathrm{Sr}_{2} \mathrm{YRu}_{1-x} \mathrm{Cu}_{x} \mathrm{O}_{6-\delta}$ $(x=0,0.1$, and 0.5$)$. Secondary phases are marked by asterisks.

\section{RESULTS AND DISCUSSION}

\section{A. $\mathrm{Sr}_{2} \mathrm{YRu}_{1-x} \mathrm{Cu}_{x} \mathrm{O}_{6-\delta}$}

Figure 1 shows the $\mathrm{X}$-ray diffraction patterns of $\mathrm{Sr}_{2} \mathrm{YRu}_{1-x} \mathrm{Cu}_{x} \mathrm{O}_{6-\delta}$ for $x=0,0.1$, and 0.5 . The major reflections can be indexed with orthorhombic symmetry. Within the instrumental resolution of a few percent, the absence of impurity lines for $x=0$ and 0.1 shows high phase purity and suggests the existence of $\mathrm{Ru}-\mathrm{Cu}$ solid solution in this doubleperovskite component. The presence of impurity peaks at $x$ $=0.5$ (marked with asterisks), on the other hand, indicates a $\mathrm{Cu}$ solubility limit below 0.5 , although it was impossible to identify the corresponding phases due to the low intensity of diffraction data. Comparing the peak intensities, we roughly estimate that the secondary phases occupy less than $20 \%$ of the volume. Least-squares fits to the Pbnm space group shows a systematic decrease of the orthorhombic distortion and an increase of the lattice parameter $c$ with the $\mathrm{Cu}$ content, i.e., $a: b: c=5.712(2): 5.773(4): 8.164(9) \AA$, $5.715(8): 5.771(8): 8.165(6) \AA$, and $5.723(1): 5.764(6): 8.1$ $\AA$ for $x=0,0.1$, and 0.5 , respectively. We have noticed slight discrepancies in lattice constants for $x=0$ in comparison with cell parameters $a=5.7690(6) \AA, b=5.7777(6) \AA$, and $c$ =8.1592(9) A published in Ref. 8. Similar discrepancies and trends in lattice constant with $\mathrm{Cu}$ content have been reported in a previous publication. ${ }^{2}$

Figure 2 shows the result of DTA analysis of multiphase $\mathrm{Sr}_{2} \mathrm{YRu}_{0.5} \mathrm{Cu}_{0.5} \mathrm{O}_{6-\delta}$ compound (powder) with increasing temperature in air. Endothermic peaks appear near 1130 and $1275^{\circ} \mathrm{C}$. The $\mathrm{Cu}$-rich sample sintered at $1360{ }^{\circ} \mathrm{C}$ became harder and looked partially melted at the bottom. The temperature dependence of the dc magnetization of $\mathrm{Sr}_{2} \mathrm{YRu}_{1-x} \mathrm{Cu}_{x} \mathrm{O}_{6-\delta}(x=0.1$ and $x=0.5)$ samples measured at $7 \mathrm{Oe}$ and the real ac susceptibility curve (at $\left.H_{d c}=0 \mathrm{Oe}, H_{a c}=3 \mathrm{Oe}\right)$ of $\mathrm{Sr}_{2} \mathrm{YRu}_{0.5} \mathrm{Cu}_{0.5} \mathrm{O}_{6-\delta}$ are presented in Fig. 3.

The general behavior of the dc magnetic susceptibility curves [Fig. 3(a)] of the $\mathrm{Sr}_{2} \mathrm{YRu}_{0.9} \mathrm{Cu}_{0.1} \mathrm{O}_{6-\delta}$ sample is very similar to that of the parent compound $\mathrm{Sr}_{2} \mathrm{YRuCuO}_{6}{ }^{8,9}$

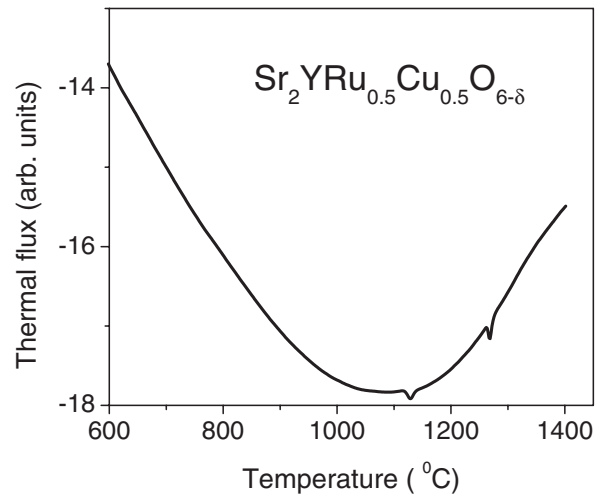

FIG. 2. DTA curves for $\mathrm{Sr}_{2} \mathrm{YRu}_{1-x} \mathrm{Cu}_{x} \mathrm{O}_{6-\delta}$ measured in an increasing temperature process in air.

The system orders AFM at $T_{N}=25-30 \mathrm{~K}$ and yet showsconspicuously weak ferromagnetism by large irreversibility. For $x=0.5$, a similar magnetic bump at $25 \mathrm{~K}$ is observed. The zero field cooled (ZFC) and field cooled (FC) curves, however, split at higher temperature $\sim 58 \mathrm{~K}$ [Fig. 3(a), inset]. These results strongly suggest that the magnetic structure remains unchanged for $\mathrm{Cu}$-doped samples and it is consistent with data published in the literature..$^{2,5,11}$ In all the samples tested, no trace of superconductivity has been observed for $x=0.1$, in rough agreement with previous reports. ${ }^{2,5,11}$ The situation is different in the case of $x=0.5$. The negative signals in the ZFC curve at $5 \mathrm{~K}$, which correspond to a screened volume fraction of $4 \%$ or larger, clearly indicate a SC component [Fig. 3(a)], which is consistent with both the negative FC magnetization around $40 \mathrm{~K}$ [inset, Fig. 3(a)] and the real

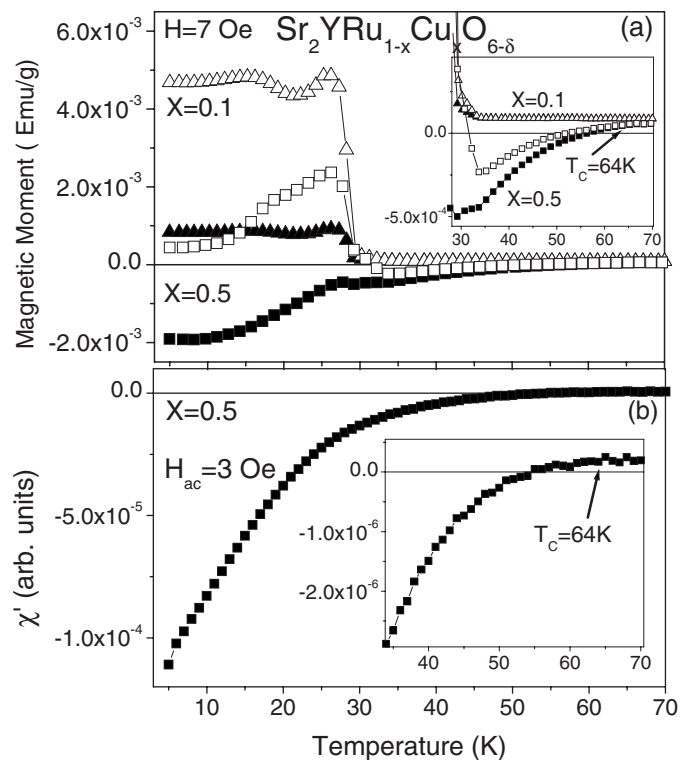

FIG. 3. (a) ZFC (solid symbols) and FC (open symbols) magnetization curves for $\mathrm{Sr}_{2} \mathrm{YRu}_{1-x} \mathrm{Cu}_{x} \mathrm{O}_{6-\delta}$ with $x=0.1$ (triangle symbols) and $x=0.5$ (square symbols). Inset: ZFC and FC magnetization curves between 28 and $70 \mathrm{~K}$. (b) The real part of the ac susceptibility (at $H_{d c}=0 \mathrm{Oe}$ and $H_{a c}=3 \mathrm{Oe}$ ) of $\mathrm{Sr}_{2} \mathrm{YRu}_{0.5} \mathrm{Cu}_{0.5} \mathrm{O}_{6-\delta}$. The inset shows the onset of superconductivity in an expanded scale. 


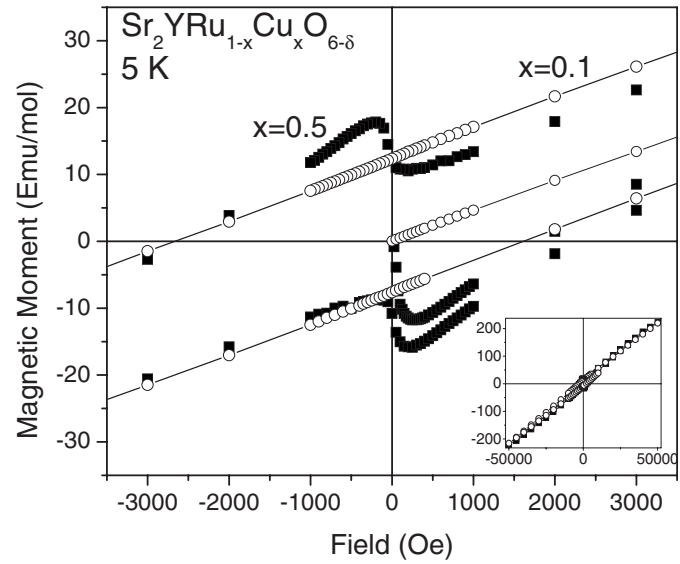

FIG. 4. $M(H)$ plot at $5 \mathrm{~K}$ for $\mathrm{Sr}_{2} \mathrm{YRu}_{1-x} \mathrm{Cu}_{x} \mathrm{O}_{6-\delta}$ with $x=0.1$ (open circles) and $x=0.5$ (solid circles); the applied fields are in the range $-3500 \mathrm{Oe}<H<3500 \mathrm{Oe}$. Inset: $M(H)$ plot in the range $-5 \mathrm{~T}<H<5 \mathrm{~T}$.

part $\chi^{\prime}$ of the ac susceptibility [Fig. 3(b)]. It is interesting to note that the onset of the SC occurs at $64 \mathrm{~K}$, as indicated by the downturns in both the FC magnetization (flux expulsion) and the $\chi^{\prime}$. The magnetization $M(H)$ plots at $5 \mathrm{~K}$ of the compounds with $x=0.1$ and 0.5 up to $3500 \mathrm{Oe}$ and $5 \mathrm{~T}$ are shown, respectively, in Fig. 4 and its inset. The virgin curve for the $x=0.5$ sample synthesized at $1360{ }^{\circ} \mathrm{C}$ clearly shows a $\mathrm{SC}$ signature. The diamagnetic signal starts decreasing above applied fields of $280 \mathrm{Oe}$, and turns to zero at $1800 \mathrm{Oe}$. The compound seems to have a lower critical field $\left(H_{c 1}\right)$ of around $280 \mathrm{Oe}$. The shielding fraction (SF) deduced from the isothermal $M(H)$ curve is $\sim 1.5 \%$ of the $-\frac{1}{4 \pi}$ value, indicating that only a small fraction of the material becomes superconducting. For the samples synthesized at $1290{ }^{\circ} \mathrm{C}$ (not shown in the figure), on the other hand, no indications of superconductivity have been noticed. Both a high doping level, i.e., above the solubility limit of $\mathrm{Cu}$, and a high synthesis temperature seem to be necessary for the appearance of superconductivity.

To explore the origin of this minor superconductivity, the grain morphology of the polished $\mathrm{Sr}_{2} \mathrm{YRu}_{0.5} \mathrm{Cu}_{0.5} \mathrm{O}_{6-\delta}$ sample, which shows the clear superconducting signal, was determined by SEM. Two types of granular structures can be clearly identified, although both have a similar grain size of 10-13 $\mu \mathrm{m}$. One type (marked as I in Fig. 5) consists of more or less round grains with many voids along the grain boundaries. This is rather similar to those of the samples synthesized at lower temperature, which is also typically characteristic of ceramics below the melting temperature. Another type (marked as II), however, consists of thick wavering sheets with well defined intergrain boundaries characterized by sharp edges. We interpret these as evidence of partial melting during synthesis. It is worth noting that there are practically no voids along the type-II grain boundaries. EDAX further reveals a distinct difference between the two types. For the type-I grains, no systematic intragrain stoichiometry variation is detected although the ratio $\mathrm{Sr}: \mathrm{Y}: \mathrm{Ru}: \mathrm{Cu}$ does vary from one grain to another: a normalized ratio of 2:1.2:1.3:0.4 represents most of the grains. Some spherical

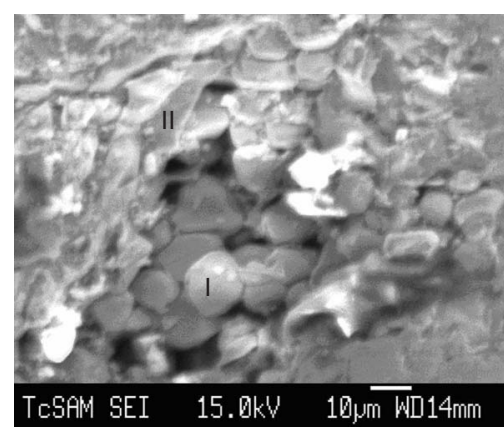

FIG. 5. SEM images of $\mathrm{Sr}_{2} \mathrm{YRu}_{0.5} \mathrm{Cu}_{0.5} \mathrm{O}_{6-\delta}$. The spherical grains with structurally intact boundaries are marked as type I. The partially melted grains without intergrain voids are marked as type II.

grains with a poor $\mathrm{Cu}$ concentration also have been observed. The typical normalized stoichiometric ratio of these particles is $2: 1.1: 1.4: 0.01$, which is similar to the structure of the parent $\mathrm{Sr}_{2} \mathrm{YRuO}_{6}$ compound. In addition to the major phase in $\mathrm{Sr}_{2} \mathrm{YRu}_{0.5} \mathrm{Cu}_{0.5} \mathrm{O}_{6-\delta}$, we found that the type-II grains correspond to a secondary phase with a general stoichiometric relation of 1.3:2:3.7:0.2 (Y:Sr:Cu:Ru), which is close to the 1:2:3 phase and consistent with YSCO. Apparently, this secondary phase for $x=0.5$ is below $5 \%$ and therefore has not been identified by XRD measurements. Detailed EDAX of the $\mathrm{Cu}$-rich type-II grains shows that deficiency in $\mathrm{Ru}$ content continuously increases with distance from the center of the grains, and almost pure YSCO structure $[1.2(\mathrm{Y}): 2(\mathrm{Sr}): 3.3(\mathrm{Cu}): 0.03(\mathrm{Ru})]$ is observed on the boundaries. Both the $\mathrm{Ru}$ depletion and redistribution occur in the melted region, while no visible deviation in $\mathrm{Y}$ and $\mathrm{Sr}$ composition appears. The observed $\mathrm{Ru}$ depletion may be due to sublimation of ruthenium oxide in the oxidizing atmosphere, ${ }^{19}$ and the $\mathrm{Ru} / \mathrm{Cu}$ redistribution will be a natural result of the correlation between the melting temperature and $\mathrm{Cu}$ doping. Thus, partially melted grains may lead to the $\mathrm{Ru}$ absence and, therefore, to the YSCO phase on the boundaries. The polycrystalline sample $\mathrm{Sr}_{2} \mathrm{YRu}_{0.5} \mathrm{Cu}_{0.5} \mathrm{O}_{6-\delta}$ was studied by Raman spectroscopy. Further verification of the presence of YSCO minority phase comes from the Raman spectra probed at type-I and type-II grains. As illustrated in Fig. 6, the spectra of the two types of grains differ significantly. While those from type I are in good agreement with the spectra of polycrystalline $\mathrm{Sr}_{2} \mathrm{YRu}_{0.9} \mathrm{Cu}_{0.1} \mathrm{O}_{6-\delta}$, published in Ref. 20, the spectra from the type-II grains are consistent with those reported earlier for YSCO. ${ }^{21}$

\section{B. Y-Sr-Cu-O system}

In an attempt to understand the appearance of YSCO under ambient pressure, we investigated the $\mathrm{Y}-\mathrm{Sr}-\mathrm{Cu}-\mathrm{O}$ system, particularly the samples with nominal compositions $\mathrm{YSrCuO}_{t}, \mathrm{YSr}_{2} \mathrm{Cu}_{2} \mathrm{O}_{t}$, and $\mathrm{Y}_{1.5} \mathrm{Sr}_{2} \mathrm{Cu}_{2} \mathrm{O}_{t}$. Superconductivity was used as the experimental indicator of the YSCO phase. In general, it is believed that pure YSCO without chemical doping can be synthesized only under high pressure in the temperature range $1050-1130{ }^{\circ} \mathrm{C} .{ }^{22-24}$ On the other hand, 


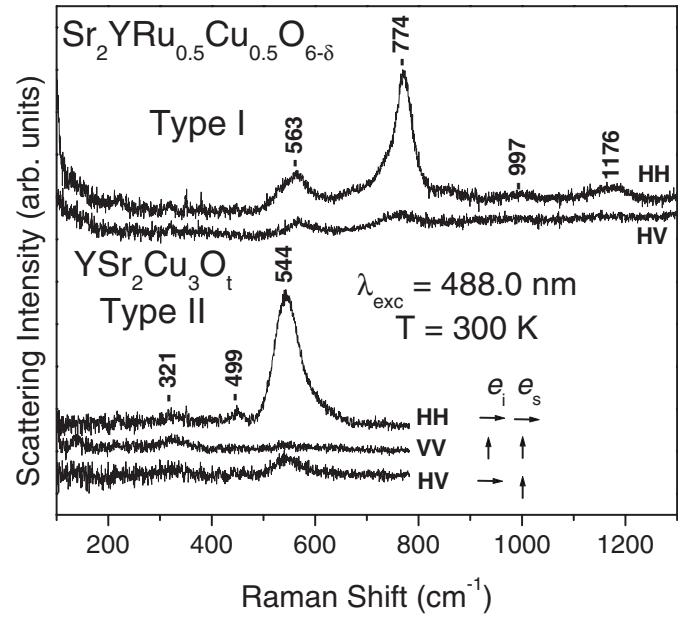

FIG. 6. Polarized Raman spectra of $\mathrm{Sr}_{2} \mathrm{YRu}_{0.5} \mathrm{Cu}_{0.5} \mathrm{O}_{6-\delta}$ (nominal composition) as obtained from type-I and type-II grains at room temperature.

trace SC has been observed in a multiphase sample with nominal composition $\mathrm{YSrCuO}_{t}$ synthesized under ambient pressure above $1200{ }^{\circ} \mathrm{C} .{ }^{18,25}$ In the absence of detailed structural analysis, the authors have tentatively assigned the $80 \mathrm{~K}$ SC phase to the YSCO and AFM transition to $\mathrm{Y}_{2} \mathrm{Cu}_{2} \mathrm{O}_{5}$ magnetic phase around $14 \mathrm{~K}$. Our magnetization data (Fig. 7) of multiphase $\mathrm{Y}-\mathrm{Sr}-\mathrm{Cu}-\mathrm{O}$ compounds closely resemble the earlier reported results. ${ }^{18,25}$ Namely, all Y-Sr-Cu-O materials sintered at $1130{ }^{\circ} \mathrm{C}$ show similar onsets of SC. The estimated SF fraction for all SC samples at $5 \mathrm{~K}$ is around $1 \%$. For samples processed at the lower temperature of $990{ }^{\circ} \mathrm{C}$, however, the SC phase did not form. The grain morphology of multiphase $\mathrm{Y}-\mathrm{Sr}-\mathrm{Cu}-\mathrm{O}$ compounds has been observed by SEM. An example of the granular structures of SC and non-SC $\mathrm{YSrCuO}_{t}$ samples is shown in Fig. 8. The changes in grain structures with the sintered temperature are well pronounced. One represents the coupling between partially melted grains [Fig. 8(a)], which are closely packed without inter-grain voids, while the other type [Fig. 8(b)] involves well-coupled grains with structurally intact boundaries. EDAX, similar to that of $\mathrm{Sr}_{2} \mathrm{YRu}_{0.5} \mathrm{Cu}_{0.5} \mathrm{O}_{6-\delta}$ samples, indi-

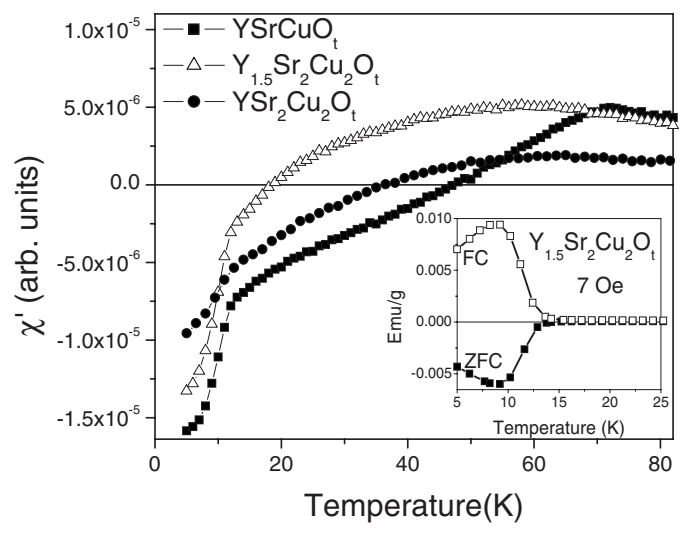

FIG. 7. The real ac susceptibility curves (at $H_{d c}=0$ Oe and $\left.H_{a c}=3 \mathrm{Oe}\right)$ of nominal compounds $\mathrm{YSrCuO}_{t}, \mathrm{YSr}_{2} \mathrm{Cu}_{2} \mathrm{O}_{t}$, and $\mathrm{Y}_{1.5} \mathrm{Sr}_{2} \mathrm{Cu}_{2} \mathrm{O}_{t}$. Inset: $\mathrm{ZFC}$ and $\mathrm{FC}$ magnetization curves of $\mathrm{Y}_{1.5} \mathrm{Sr}_{2} \mathrm{Cu}_{2} \mathrm{O}_{t}$ (nominal composition) at applied field $7 \mathrm{Oe}$.

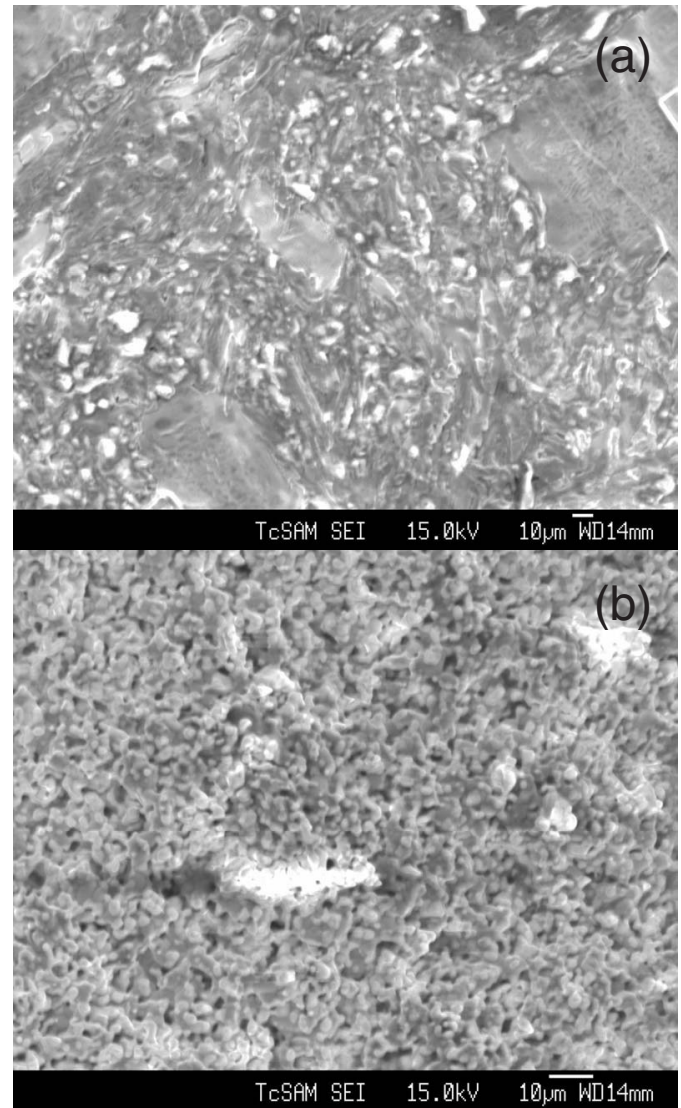

FIG. 8. SEM image of (a) SC (sintered at $1130{ }^{\circ} \mathrm{C}$ ) and (b) non-SC (sintered at $990{ }^{\circ} \mathrm{C}$ ) $\mathrm{YSrCuO}_{t}$ samples (nominal composition).

cates the presence of the YSCO structure only in samples sintered at high temperature. The internal stresses during the solidification, therefore, may offer a natural interpretation for the formation of the YSCO phase.

\section{CONCLUSIONS}

We have shown that superconductivity in $\mathrm{Sr}_{2} \mathrm{YRu}_{0.5} \mathrm{Cu}_{0.5} \mathrm{O}_{6-\delta}$ and various $\mathrm{Y}-\mathrm{Sr}-\mathrm{Cu}-\mathrm{O}$ systems appears only in partially melted samples under ambient pressure. The superconducting behavior is tentatively attributed to a minor $\mathrm{YSr}_{2} \mathrm{Cu}_{3} \mathrm{O}_{t}$ phase, which occurs on grain boundaries in the first system due to the depletion and redistribution of $\mathrm{Ru}$ in partially melted grains, and occurs in the latter system due to the internal stress during solidification.

\section{ACKNOWLEDGMENTS}

The work in Houston is supported in part by the T. L. L. Temple Foundation, the John J. and Rebecca Moores Endowment, and the State of Texas through the Texas Center for Superconductivity at the University of Houston, and at Lawrence Berkeley Laboratory by the Director, Office of Science, Office of Basic Energy Sciences, Division of Materials Sciences and Engineering of the U.S. Department of Energy under Contract No. DE-AC03-76SF00098. 
${ }^{1}$ Y. Maeno, H. Hashimoto, K. Yoshida, S. Nishizaki, T. Fujita, J. G. Bednorz, and F. Lichtenberg, Nature (London) 372, 532 (1994).

${ }^{2}$ M. K. Wu, D. Y. Chen, F. Z. Chien, S. R. Sheen, D. C. Ling, C. Y. Tai, G. Y. Tseng, D. H. Chen, and F. C. Zhang, Z. Phys. B: Condens. Matter 102, 37 (1997).

${ }^{3}$ H. A. Blackstead, J. D. Dow, D. R. Harshman, M. J. DeMarco, M. K. Wu, D. Y. Chen, F. Z. Chien, D. B. Pulling, W. J. Kossler, A. J. Greer, C. E. Stronach, E. Koster, B. Hitti, M. Haka, and S. Toorongian, Eur. Phys. J. B 15, 649 (2000).

${ }^{4}$ J. D. Dow, H. A. Blackstead, and D. R. Harshman, Physica C 364-365, 74 (2001).

${ }^{5}$ H. A. Blackstead, J. D. Dow, D. R. Harshman, W. B. Yelon, M. X. Chen, M. K. Wu, D. Y. Chen, F. Z. Chien, and D. B. Pulling, Phys. Rev. B 63, 214412 (2001).

${ }^{6}$ P. C. Donohue and E. L. McCann, Mater. Res. Bull. 12, 519 (1977).

${ }^{7}$ R. Greatrex, N. N. Greenwood, M. Lal, and I. Fernandez, J. Solid State Chem. 30, 137 (1979).

${ }^{8}$ P. D. Battle and W. J. Macklin, J. Solid State Chem. 54, 245 (1984).

${ }^{9}$ G. Cao, Y. Xin, C. S. Alexander, and J. E. Crow, Phys. Rev. B 63, 184432 (2001).

${ }^{10}$ J. Dow and D. Harshman, Int. J. Mod. Phys. B 17, 3310 (2003).

${ }^{11}$ D. R. Harshman, W. J. Kossler, A. J. Greer, D. R. Noakes, C. E. Stronach, E. Koster, M. K. Wu, F. Z. Chien, J. P. Franck, I. Isaac, and J. D. Dow, Phys. Rev. B 67, 054509 (2003).

${ }^{12}$ J. D. Dow, H. A. Blackstead, Z. F. Ren, and D. Z. Wang, JETP
Lett. 80, 190 (2004).

${ }^{13}$ C. Bernhard, J. L. Tallon, Ch. Niedermayer, Th. Blasius, A. Golnik, E. Brucher, R. K. Kremer, D. R. Noakes, C. E. Stronach, and E. J. Ansaldo, Phys. Rev. B 59, 14099 (1999).

${ }^{14}$ W. E. Pickett, R. Weht, and A. B. Shick, Phys. Rev. Lett. 83, 3713 (1999).

${ }^{15}$ B. Lorenz, R. L. Meng, J. Cmaidalka, Y. S. Wang, J. Lenzi, Y. Y. Xue, and C. W. Chu, Physica C 363, 251 (2001).

${ }^{16}$ P. W. Klamut, B. Dabrowski, S. Kolesnik, M. Maxwell, and J. Mais, Phys. Rev. B 63, 224512 (2001).

${ }^{17}$ T. Motohashi, A. Yoshikawa, J. Shimoyama, and K. Kishio, Physica C 282-287, 515 (1997).

${ }^{18}$ M. K. Wu, J. R. Ashburn, C. A. Higgins, B. H. Loo, D. H. Burns, A. Ibrahim, T. D. Rolin, F. Z. Chien, and C. Y. Huang, Phys. Rev. B 37, 9765 (1988).

${ }^{19}$ C. A. Georg, P. Triggs, and F. Levy, Mater. Res. Bull. 17, 105 (1982).

${ }^{20}$ H. L. Liu, J. L. Her, C. C. Chen, S. M. Rao, M. K. Wu, W. F. Hsueh, C. C. Chi, and F. Z. Chien, J. Phys. Chem. Solids 67, 302 (2006).

${ }^{21}$ H.-G. Lee, A. P. Litvinchuk, M. V. Abrashev, M. N. Iliev, S. H. $\mathrm{Xu}$, and C. W. Chu, J. Phys. Chem. Solids 59, 1994 (1998).

${ }^{22}$ B. Okai, Jpn. J. Appl. Phys., Part 2 29, L2180 (1990).

${ }^{23}$ A. Ono, Physica C 198, 287 (1992).

${ }^{24}$ E. Gilioli, P. G. Radaelli, A. Gauzzi, F. Licci, and M. Marezio, Physica C 341-348, 605 (2000).

${ }^{25}$ R. Suryanarayanan, S. B. Baliga, A. L. Jain, and O. Gorochov, Mod. Phys. Lett. B 4, 159 (1990). 